\title{
A CLASS OF NONCOMMUTATIVE POWER-ASSOCIATIVE ALGEBRAS $\left({ }^{1}\right)$
}

\author{
BY \\ ROBERT H. OEHMKE
}

1. Introduction. Let $A$ be a nonassociative algebra over a field $F$. If for each $x$ in $A$ the subalgebra $F[x]$ of all polynomials in $x$ over $F$ is an associative algebra we call $A$ a power-associative algebra. In particular the elements of $A$ must satisfy the identity

$$
x \cdot(x \cdot x)=(x \cdot x) \cdot x .
$$

To any algebra $A$ over a field $F$ of characteristic not 2 we can attach a commutative algebra $A^{(+)}$over $F$ which is the same vector space as $A$ and which has a product $(x, y)$ expressible in terms of the product $x \cdot y$ of $A$ by

$$
(x, y)=\frac{1}{2}(x \cdot y+y \cdot x) .
$$

Powers of elements in $A^{(+)}$agree with their powers in $A$. Thus the powerassociativity of $A$ implies the power-associativity of $A^{(+)}$.

This paper is chiefly concerned with the class of nonassociative algebras satisfying (1) and having $A^{(+)}$a separable Jordan algebra. We shall denote this class by the letters $S J$.

Suppose that $G$ is a nonassociative algebra over a field $F$ of characteristic not 2 and $G$ contains a subspace $S$ closed under the operation $(x, y)$ defined by (2). The set of all finite linear combinations of elements in $G$ of the form $x y-y x$ for $x$ and $y$ in $S$ form a subspace $U(S)$ of $G$. If $T$ is any linear mapping on $L(S)$ in to $S$ we call the mapping $T$ a bonding mapping of $G[2]\left({ }^{2}\right)$. Every bonding mapping determines an algebra $B(G, S, T)$ which is the same vector space as $S$ and is defined by the product

$$
x \cdot y=\frac{1}{2}(x y+y x)+(x y-y x) T
$$

for all $x$ and $y$ in $S$, where $x y$ is the product in $G$. We say that $B(G, S, T)$ is bonded to $G$. We note that

Received by the editors September 17, 1956.

(1) This paper is a part of the author's discertation submitted in partial fulfilment of the requiremente of the degree of J)octor of Philosophy at the University of Chicago. The author is indebted to Professor $A$. A. Albert who directed the progress of this dissertation.

(2) Numbers in brackets refer to the bibliography. 


$$
x \cdot y+y \cdot x=x y+y x .
$$

Albert has shown [2] that any algebra of characteristic not 2, 3 or 5, satisfying (1) and having $A^{(+)}$a central simple Jordan algebra of degree $t>2$ has a scalar extension $A_{K}$ which is obtained from a bonding mapping. Here the degree $t$ is defined as the maximum number of primitive orthogonal idempotents $e_{i}$ in the expression of the unity quantity $e=e_{1}+\cdots+e_{t}$ for any scalar extension of the algebra. We shall extend this result and show that for all algebras $A$ of class $S J$ there exists a direct sum $G$ of simple associative algebras and 3 by 3 matric algebras over a Cayley algebra such that $A$ $=B(G, S, T)$.

A class of algebras $A$ satisfying (1) such that $A^{(+)}$is a semi-simple Jordan algebra with an inseparable component will be exhibited for which no bonding mapping exists.

2. Bonding mappings. A separable Jordan algebra is defined as a semisimple Jordan algebra each of whose components has a separable center over its base field. Let $A$ be an algebra of class $S J$ over the field $F$. The algebra $A^{(+)}$is a semi-simple Jordan algebra over $F$ and is therefore a direct sum of simple Jordan algebras.

Any simple Jordan algebra is isomorphic to a Jordan algebra of linear transformations or is an algebra of order 27 over its center. These algebras are called special and exceptional Jordan algebras respectively. The simple special Jordan algebras are the Jordan algebras associated with one of the following types under the multiplication defined in (2): (a) a simple associative algebra over $F$, (b) the set of symmetric elements of a simple associative algebra with an involution $J$ of the first kind over $F$, (c) the set of symmetric elements of a simple associative algebra with an involution $J$ of the second kind over $F$, (d) a Clifford system over a field $K$ containing $F[1 ; 3 ; 5]$.

Theorem 1. Let $A$ be an algebra over a field $F$ of class $S J$ and let $A^{(+)}$be special. Then there exists an associative algebra $G$ over $F$ such that $A=B(G, S, T)$.

We first prove the following elementary results.

Lemma 1. If for some scalar extension $K$ of $F$ we have $A_{K}$ bonded to $G_{K}$ the algebra $A$ is bonded to $G$.

Let $T_{0}$ be a linear mapping satisfying (3) for all $x$ and $y$ in $A_{K}$. Then in particular if $x$ and $y$ are in $A$ we have $x y-y x$ in $U\left(A_{K}\right)$ and $(x y-y x) T_{0}$ $=x \cdot y-(x y+y x) / 2$. Since $x \cdot y$ and $x y+y x=x \cdot y+y \cdot x$ are in $A$ we also have $(x y-y x) T_{0}$ in $A$. The transformation $T_{0}$ cut down to $A$ induces a linear mapping $T$ over $F$ such that $(x y-y x) T_{0}=(x y-y x) T$ for all $x$ and $y$ in $A$. The mapping $T$ is the desired mapping bonding $A$ to $G$.

Now let $A=A_{1}+\cdots+A_{n}$ where each $A_{r}^{(+)}$is an ideal of $A^{(+)}$and a simple Jordan algebra. Even though each $A_{r}^{(+)}$is an ideal of $A^{(+)}$it doesn't necessarily follow that $A_{r}$ is an ideal of $A$ or even a subalgebra of $A$ under the 
multiplication $x \cdot y$. However we do have the following:

Lemma 2. If $x$ is in $A_{i}, y$ is in $A_{j}$ and $i \neq j$ we have $x \cdot y=0$.

By a linearization process and (4), (1) becomes

$$
\begin{aligned}
x \cdot(y z+z y)+y \cdot(x z+z x) & +z \cdot(x y+y x) \\
& =(y z+z y) \cdot x+(x z+z x) \cdot y+(x y+y x) \cdot z .
\end{aligned}
$$

If we let $z=e_{j}$ in (5) where $e_{j}$ is the unity element of $A_{j}$ we obtain $x \cdot y=y \cdot x$. From (4) we have $2 x \cdot y=x y+y x=0$, so $x \cdot y=0$.

We have seen that the only nonzero products in $A$ arise from the products of two elements of the same component of $A^{(+)}$. Therefore we need only show the existence of an algebra $G_{r}$, a subspace $S_{r}$ of $G_{r}$ equal to the space $A_{r}$, and a transformation $T_{r}$ mapping $U\left(S_{r}\right)$ into $A$ and satisfying (3) for all elements $x$ and $y$ in $A_{r}$. The composition of all the $G_{r}, S_{r}$ and $T_{r}$ will be the desired $G, S$ and $T$. The $G_{r}, S_{r}$ and $T_{r}$ will be defined separately for each of the types (a)-(d) above.

If $A_{r}^{(+)}$is of type (a), it is the Jordan algebra associated with a simple separable associative algebra $C$ over $F$. Since $C$ is separable it has a splitting field $K$. The extension $C_{K}$ is a direct sum of total matric algebras. By Lemma 1 and Lemma 2 we can assume that $C$ is a total matric algebra and $A_{r}^{(+)}$is the Jordan algebra associated with $\mathrm{kt}$. The algebra has a matric basis $\left(\cdots e_{i j} \cdots\right)$ over $F$.

Lemma 3. If $A_{r}^{(+)}$is the Jordan algebra associated with the total matric algebra $C$ then $G_{r}=S_{r}=C$ and $T_{r}$ is defined by

$$
\begin{array}{ll}
\left(e_{i j}\right) T_{r}=e_{i i} \cdot e_{i j}-e_{i j} / 2 & \text { for } i \neq j, \\
\left(e_{i i}\right) T_{r}=-e_{1 i} \cdot e_{i 1}+e_{i i} / 2 . &
\end{array}
$$

Since (3) is linear over $F$ we need only show that it is satisfied for the basal elements or that

$$
e_{i j} \cdot e_{p q}=\frac{1}{2} e_{i j} \cdot e_{p q}+\frac{1}{2} e_{p q} \cdot e_{i j}+\left(e_{i j} e_{p q}-e_{p q} e_{i j}\right) T_{r} .
$$

To prove that (7) holds under the definition in (6) we assume first that $i \neq j, p, q$ and $j \neq p, q$. Put $x=e_{i j}, y=e_{p q}$ and $z=e_{p p}$ in (5). We obtain $e_{i j} \cdot e_{p q}$ $=e_{p q} \cdot e_{i j}$. This identity together with (4) gives us $e_{i j} \cdot e_{p q}=0$. Since the right hand member of (7) is 0 it has been verified in this case. Assume next that $i=p \neq q, j$. We put $x=e_{i j}, y=e_{i j}, z=e_{j q}$ in (5) and obtain $e_{i j} \cdot e_{i q}=0$ and again (7) is satisfied. Now if $j=p$ and $i=q$ we must show that

$$
e_{i j} \cdot e_{j i}=e_{i i}-e_{1 i} \cdot e_{i 1}+e_{1 j} \cdot e_{j 1} \text {. }
$$

We use (5) with $x=e_{i j}, y=e_{j 1}$ and $z=e_{1 i}$, all indices distinct, and obtain (8). If $i=j$, (8) reduces to $e_{i i} \cdot e_{i i}=e_{i i}$ which is clear from (4). If $i=1$ in (8) this 
becomes $e_{1 j} \cdot e_{j 1}=e_{1 j} \cdot e_{j 1}$. If $i=j$ and $i \neq p=q$ in (7) the right hand member is zero. This identity is satisfied if $e_{i i} \cdot e_{p p}$ is also zero. The product $e_{i i} \cdot e_{p p}$ is seen to be 0 when we put $x=e_{i i}, z=y=e_{p p}$ in (5). The only remaining case to consider is $i=q$ and $j \neq p$. We need to show that $e_{i j} \cdot e_{p i}=e_{p j}-e_{p p} \cdot e_{p j}$ which is clear from (5) with $x=e_{i j}, y=e_{p i}$ and $z=e_{p p}$. Thus (3) is satisfied for $x$ and $y$ and $A_{r}$ and $T_{r}$ defined as above.

If $A_{r}^{(+)}$is of type (b) it is the Jordan algebra associated with the set of symmetric elements of a simple separable associative algebra $C$ over $F$ with an involution $J$ over $F$ of the first kind. If $K$ is a splitting field of $C$ the involution $J$ can be extended uniquely to an involution over $K$ of $C_{K}$. The algebra $C_{K}$ is a direct sum of total matric algebras with an involution $J_{0}$. The set of symmetric elements of $C_{K}$ is $\left(A_{r}^{(+)}\right)_{K}$. By Lemma 1 we may assume that $F=K$. We need the following two lemmas on involutions.

Lemma 4. If $J$ is an involution of a direct sum of total matric algebras any component $M$ is left fixed by $J$ or is mapped into a component $M^{J}$ of the same degree. The total matric algebra $M^{J}$ will have a matric basis $\left(\cdots f_{i j} \cdots\right)$ where $f_{i j}=e_{j i}^{J}$ and $\left(\cdots e_{j i} \cdots\right)$ is a matric basis of $M$.

We shall omit the proof of this lemma.

If $M$ is a total matric algebra of degree $n=2 m$ then $M=M_{m} \times M_{2}$ (direct product) where $M_{2}=\left(g_{11}, g_{12}, g_{21}, g_{22}\right)$ and $M_{m}=\left(\cdots e_{i j} \cdots\right)$ for $i, j$ $=1, \cdots, m$. Every element of $M$ is uniquely expressible in the form

$$
a=A g_{11}+B g_{12}+C g_{21}+D g_{22}
$$

where $A, B, C$ and $D$ are elements of $M_{m}$. If we define

$$
a^{J}=D^{\prime} g_{11}-B^{\prime} g_{12}-C^{\prime} g_{21}+A^{\prime} g_{22}
$$

where $A^{\prime}, B^{\prime}, C^{\prime}$ and $D^{\prime}$ are the transposes of $A, B, C$ and $D . J$ is an involution of $M$. The set of symmetric elements of $M$ are of the form $a=A g_{11}$ $+\left(B-B^{\prime}\right) g_{12}+\left(C-C^{\prime}\right) g_{21}+A^{\prime} g_{22}[4]$.

Two involutions $J$ and $J^{\prime}$ of an algebra $C$ are called cogredient if there exists an automorphism $S$ over the center of $C$ such that $J^{\prime}=S^{-1} J S$.

Lemma 5. An involution $J$ of a total matric algebra $M$ is cogredient to transposition or to the involution described in (9)-(10).

The proof of this lemma can be found in [4].

By these two lemmas we see that $J_{0}$ leaves $M$ fixed and is cogredient to transposition, $J_{0}$ leaves $M$ fixed and is cogredient to the involution defined in (9)-(10) or $J_{0}$ maps $M$ into an isomorphic component. We shall treat each of these separately.

We first assume that $J_{0}$ is cogredient to transposition and leaves $M$ fixed. We can choose a matric basis $\left(\cdots e_{i j} \cdots\right)$ on $M$ such that $e_{i j}^{J}=e_{j i}$.

Lемма 6. If $A_{r}^{(+)}$is the Jordan algebra associated with the set of symmetric 
elements under transposition of a total matric algebra $M$ then $G_{r}=M, S_{r}=$ set of symmetric elements and $T_{r}$ is defined as

$$
\left(e_{i j}-e_{j i}\right) T_{r}=-e_{j j} \cdot\left(e_{i j}+e_{j i}\right)+\frac{1}{2}\left(e_{i j}+e_{j i}\right) \quad \text { for } i \neq j,
$$

$$
\left(e_{i i}\right) T_{r}=\frac{1}{2} e_{i i}
$$

To prove that $T_{r}$ satisfies (3) we need only show that it is satisfied for the elements $e_{i i}$ and $e_{i j}+e_{j i}$ or that

$$
\begin{aligned}
\left(e_{i j}+e_{j i}\right) \cdot\left(e_{p q}+e_{q p}\right)= & \frac{1}{2}\left(e_{i j}+e_{j i}\right)\left(e_{p q}+e_{q p}\right)+\frac{1}{2}\left(e_{p q}+e_{q p}\right)\left(e_{i j}+e_{j i}\right) \\
& +\left[\left(e_{i j}+e_{j i}\right)\left(e_{p q}+e_{q p}\right)-\left(e_{q p}+e_{p q}\right)\left(e_{i j}+e_{j i}\right)\right] T_{r} .
\end{aligned}
$$

Assume first that $i \neq p, q, j$ and $j \neq p, q$. Put $x=e_{i j}+e_{j i}, y=e_{p q}+e_{q p}$ and $z=e_{p p}$ in (5). We obtain $\left(e_{i j}+e_{j i}\right) \cdot\left(e_{p q}+e_{q p}\right)=0$ and since the right hand side of (12) is also 0 this identity is satisfied. Next if we put $x=e_{i j}+e_{j i}, y=e_{i p}+e_{p i}$ and $z=e_{p p}$ for $p \neq i, j$ we have $\left(e_{i j}+e_{j i}\right) \cdot\left(e_{i p}+e_{p i}\right)=\left(e_{j p}+e_{p j}\right) \cdot e_{p p}=-e_{p p}$ $\cdot\left(e_{j p}+e_{p j}\right)+\left(e_{p j}+e_{j p}\right)$ when $i \neq j$ and $e_{i i} \cdot\left(e_{i p}+e_{p i}\right)=\left(e_{i p}+e_{p i}\right) \cdot e_{p p}=-e_{p p}$ $\cdot\left(e_{i p}+e_{p i}\right)+\left(e_{i p}+e_{p i}\right)$ when $i=j$ and again (12) is satisfied. Now let $x=y=e_{i i}$, $z=e_{p q}+e_{q p}$ and $i, p$ and $q$ be distinct. Identity (5) gives us $e_{i i} \cdot\left(e_{p q}+e_{q p}\right)=0$. If we put $i=p$ and $j=q$ in (12) it reduces to $\left(e_{i j}+e_{j i}\right) \cdot\left(e_{i j}+e_{j i}\right)=\left(e_{i j}+e_{j i}\right)$ $\cdot\left(e_{i j}+e_{j i}\right)$ and is certainly satisfied. It remains only to show that $e_{i i} \cdot e_{p p}=0$ when $i \neq p$. This is obtained from (5) by putting $x=y=e_{i i}$ and $z=e_{p p}$.

We next consider the case where $J_{0}$ leaves $M$ fixed and is cogredient to the involution described in (9)-(10). We shall use the following notation: $h_{i j}$ $=e_{i j} g_{11}+e_{j i} g_{22}, f_{i j}=\left(e_{i j}-e_{j i}\right) g_{12}, d_{i j}=\left(e_{i j}-e_{j i}\right) g_{21}, \bar{h}_{i j}=e_{i j} g_{11}-e_{j i} g_{22}, \bar{f}_{i j}$ $=\left(e_{i j}+e_{j i}\right) g_{12}$ and $\bar{d}_{i j}=\left(e_{i j}+e_{j i}\right) g_{21}$. The set $S_{r}$ of all $J_{0}$-symmetric elements has a basis $\left(\cdots h_{i j} \cdots f_{m n} \cdots d_{s t} \cdots\right)$. The set $U\left(S_{r}\right)$ has a basis $\left(\cdots \bar{h}_{i j} \cdots \bar{f}_{m n} \cdots \bar{d}_{s t} \cdots\right)$.

Lemma 7. If $A_{r}^{(+)}$is the Jordan algebra associated with the set of symmetric elements under the involution described in (9)-(10) of a total matric algebra $M$ then $G_{r}=M, S_{r}=$ set of symmetric elements and $T_{r}$ is defined by

$$
\begin{aligned}
\left(\bar{h}_{i j}\right) T_{r} & =-h_{j j} \cdot h_{i j}+\frac{1}{2} h_{i j}, \\
2\left(\bar{h}_{i i}\right) T_{r} & =f_{i j} \cdot d_{j i}+h_{i j} \cdot h_{j i}-h_{i i}-h_{j j}, \\
\left(\bar{f}_{i j}\right) T_{r} & =-h_{j j} \cdot f_{i j}+\frac{1}{2} f_{i j}, \\
\left(\bar{f}_{i i}\right) T_{r} & =f_{j i} \cdot h_{i j}, \\
\left(\bar{d}_{i i}\right) T_{r} & =-d_{j i} \cdot h_{j i},
\end{aligned}
$$




$$
\left(\bar{d}_{i j}\right) T_{r}=h_{i i} \cdot d_{i j}-\frac{1}{2} d_{i j}
$$

and $i \neq j$.

For a number of choices of $x$ and $y$ we have both $x y-y x$ and $x y+y x$ equal to 0 . To show that (3) is satisfied in these cases we have merely to show that $x \cdot y=0$. This can be done by using (5) with $z=h_{r r}$ and a proper choice of $r$. If both $x$ and $y$ are equal to $h_{i i}$ we have $x y-y x=0$ and $x y+y x=2 h_{i i}$, however it is easily demonstrated from (4) that $h_{i i} \cdot h_{i i}=h_{i i}$. To prove that (3) holds under the definition of $T_{r}$ in (13a) we make use of the two identities $h_{i j} \cdot h_{j q}$ $=h_{i i} \cdot h_{i q}$ and $h_{i i} \cdot h_{i q}=-h_{q q} \cdot h_{i q}+h_{i q}$ obtained from (5) by substituting $x=h_{i j}, y=h_{j i}, z=h_{i q}$ and $x=h_{i q}, y=h_{i q}$ and $z=h_{q i}$. The remaining products $x \cdot y$ with $x y-y x=\bar{h}_{i j}$ that we need to consider are $f_{i j} \cdot d_{j p}, f_{i q} \cdot d_{p q}, f_{i j} \cdot d_{p i}$ and $f_{i p} \cdot d_{i q}$. For each of these products we use (5) with $z=h_{p p}$.

In considering (13b) we must first show that the right hand member is independent of the choice of $j$. We let $x=f_{p j}, y=h_{i p}$ and $z=d_{i j}$ in (5) and get $f_{p j} \cdot d_{p j}-h_{p j} \cdot h_{j p}-f_{i j} \cdot d_{i j}+h_{i j} \cdot h_{j i}=h_{p i} \cdot h_{i p}+h_{j p} \cdot h_{p j}+h_{i j} \cdot h_{j i}-h_{i i}-h_{p p}-h_{j j}=0$. Use (5) with $x=f_{i i}, y=h_{i j}$ and $z=d_{i i}$ to obtain $-f_{i j} \cdot d_{i j}=f_{i j} \cdot d_{j i}$. By setting $x=f_{i i}, y=h_{j i}$ and $z=d_{i j}$ we obtain a third identity $f_{i j} \cdot d_{i j}=f_{i j} \cdot d_{j i}$. These three identities combine to give us $f_{i j} \cdot d_{j i}+h_{i j} \cdot h_{j i}-f_{i p} \cdot d_{p i}-h_{i p} \cdot h_{p i}-h_{j j}+h_{p p}=0$ and the right hand member of (13b) is independent of the choice of $j$. From these identities we can also obtain $\left(\bar{h}_{i i}-\bar{h}_{j j}\right) T_{r}=2^{-1} f_{i j} \cdot d_{j i}+2^{-1} h_{i j} \cdot h_{j i}-2^{-1} h_{i j}$ $\cdot h_{j i}=h_{i j} \cdot h_{j i}$ and $\left(\bar{h}_{i i}+\bar{h}_{j j}\right) T_{r}=2^{-1} f_{i j} \cdot d_{j i}+2^{-1} f_{j i} \cdot d_{i j}=f_{i j} \cdot d_{j i}$.

To prove that (3) holds with the definition of $T_{r}$ given in (13c) we let $z=h_{p p}$ and $x$ and $y$ equal the two factors of the product $x \cdot y=f_{i q} \cdot h_{p q}, f_{p q} \cdot h_{q q}$, $f_{q i} \cdot h_{p i}$ in (5). This gives us three of the necessary four identities. The fourth identity, $f_{i j} \cdot h_{i i}=h_{j j} \cdot f_{i j}$ is obtained from (5) by letting $y=h_{i i}, x=f_{i j}$ and $z=h_{j j}$.

The right hand member of (13d) is independent of the choice of $j$, for if we let $x=f_{j i}, y=h_{p j}$ and $z=h_{i p}$ in (5) we have $f_{j i} \cdot h_{i j}=f_{p i} \cdot h_{i p}$. Terms of the form $\bar{f}_{i i}$ will arise only when we consider the products $f_{i j} \cdot h_{j i}$. Clearly these products satisfy (3) as $T_{r}$ is defined in (13d) since $f_{i j} \cdot h_{j i}+h_{j i} \cdot f_{i j}=0$.

We can show that (3) holds under the definitions (13e) and (13f) by using the identities proven for (13c) and (13d) with the subscripts 1 and 2 of $g_{i j}$ interchanged and with the matric coefficients of $g_{i j}$ replaced by their transposes. This leaves $h_{i j}$ fixed and replaces $f_{i j}$ by $d_{j i}$.

Finally if $M$ is mapped onto the component $M^{J_{0}}$ distinct from $M$ by the involution $J_{0}$ the symmetric elements are linear combinations of elements $e_{i i}+f_{i i}$ and $e_{i j}+f_{j i}$ described in Lemma 4 . We have

Leмма 8. If $A_{r}^{(+)}$is the Jordan algebra associated with the set of symmetric elements under the involution $J_{0}$ of the direct sum of total matric algebras $M$ and $M^{J_{0}}$ then $G_{r}=M \oplus M^{J 0}, S_{r}=$ set of symmetric elements and $T_{r}$ is defined by 


$$
\begin{aligned}
& \left(e_{i j}\right) T_{r}=\frac{1}{2}\left(e_{i i}+f_{i i}\right) \cdot\left(e_{i j}+f_{j i}\right)-\frac{1}{2} e_{i j}, \\
& \left(f_{j i}\right) T_{r}=-\frac{1}{2}\left(e_{i i}+f_{i i}\right) \cdot\left(e_{i j}+f_{j i}\right)+\frac{1}{2} f_{j i}, \\
& \left(e_{i i}\right) T_{r}=-\frac{1}{2}\left(e_{1 i}+f_{i 1}\right) \cdot\left(e_{i 1}+f_{1 i}\right)+\frac{1}{2} e_{i i}, \\
& \left(f_{i i}\right) T_{r}=\frac{1}{2}\left(e_{1 i}+f_{i 1}\right) \cdot\left(e_{i 1}+f_{1 i}\right)-\frac{1}{2} f_{i i},
\end{aligned}
$$

and $i \neq j$.

To show that (3) is satisfied under this definition of $T_{r}$ for all products $x \cdot y$, except the product $\left(e_{i j}+f_{j i}\right) \cdot\left(e_{j i}+f_{i j}\right)$, we use (4) and (5) with $z=e_{p p}+f_{p p}$ and the proper choice of $p$. When $1 \neq i, j$ we put $x=\left(e_{i j}+f_{j i}\right), y=\left(e_{j 1}+f_{1 j}\right)$ and $z=\left(e_{1 i}+f_{i 1}\right)$ in (5) to obtain $\left(e_{i j}+f_{j i}\right) \cdot\left(e_{j i}+f_{i j}\right)=\left(e_{1 j}+f_{j 1}\right) \cdot\left(e_{j 1}+f_{1 j}\right)-\left(e_{1 i}+f_{i 1}\right)$ $\cdot\left(e_{i 1}+f_{1 i}\right)+e_{i i}+f_{i i}$. When $i=1$ (3) reduces to $\left(e_{1 j}+f_{j 1}\right) \cdot\left(e_{j 1}+f_{1 j}\right)=\left(e_{1 j}+f_{j 1}\right)$ - $\left(e_{j 1}+f_{1 j}\right)$. This completes the study of algebras of type (b).

If $A_{r}^{(+)}$is a Jordan algebra of type (c) it is the algebra over $F$ of all $J$ symmetric elements of a simple associative algebra $C$ where $J$ is an involution of the second kind. If $K$ splits $C$ over $F$ then $J$ can be extended uniquely to an involution of the second kind on $C_{K}$. The following lemma tells us the nature of this involution [3].

Lemma 9. Let $M$ be a direct sum of total matric algebras over $K$. If $M$ has an involution $J$ of the second kind over $K$ then $M$ has $2 t$ components and $J$ leaves no component fixed.

Since $J$ carries each component into an isomorphic component we may apply Lemma 8.

If $A_{r}^{(+)}$is a Jordan algebra of type (d) it is the Jordan algebra associated with a Clifford system over a field containing $F$. Again with the assumption of separability we can make a scalar extension by $K$ that splits the center of $A_{r}^{(+)}$. Then $\left(A_{r}^{(+)}\right)_{K}$ will be a direct sum of Jordan algebras associated with a Clifford system over $K$. We can assume that $F=K$ and $A_{r}^{(+)}$is one of these components by Lemmas 1 and $2 . A_{r}^{(+)}$is a subspace $\left(s_{1}, \cdots, s_{n}\right)$ of an associative algebra $C$. The multiplication of these basal elements in $C$ is given by $s_{i}^{2}=\alpha_{i} \neq 0$ in $F$ and $s_{i} s_{j}=-s_{j} s_{i}$ for $i \neq j$.

Lemma 10. If $A_{r}^{(+)}$is the Jordan algebra associated with the Clifford system $\left(s_{1}, \cdots, s_{n}\right)$ of an associative algebra $C$ then we let $G_{r}=C, S_{r}=\left(s_{1}, \cdots, s_{n}\right)$ and $T_{r}$ be defined as

$$
2\left(s_{i} s_{j}\right) T_{r}=s_{i} \cdot s_{j}
$$

To show that $T_{r}$ satisfies (3) we need only show that $s_{i} \cdot s_{i}=\alpha_{i}$ since $s_{i} \cdot s_{j}$ 
$=2^{-1} s_{i} s_{j}+2^{-1} s_{j} s_{i}+\left(s_{i} s_{j}-s_{j} s_{i}\right) T_{r}$ by the definition of $T_{r}$. Identity (4) gives us $s_{i} \cdot s_{i}+s_{i} \cdot s_{i}=2 \alpha_{i}$ so $s_{i} \cdot s_{i}=\alpha_{i}$. This completes the proof of Lemma 10 and Theorem 1.

3. The exceptional simple Jordan algebra. Let $A$ be an algebra over $F$ satisfying (1) such that $A^{(+)}$is an exceptional simple Jordan algebra whose center is separable over $F$. The algebra $A$ belongs to the class $S J$. We can make a scalar extension $K$ of $F$ such that $\left(A^{(+)}\right)_{K}$ will be a direct sum of exceptional central simple Jordan algebras over $K$. It has been shown [6] that any exceptional central simple Jordan algebra arises in the following manner. Let $G$ be the algebra of all three-rowed matrices with elements in a Cayley algebra $C$ over $K$. The algebra $C$ has a basis $\left(e, u_{2}, \cdots, u_{8}\right)$ and multiplication is defined by $e u_{i}=u_{i} e=u_{i},-u_{j} u_{j}=u_{j} u_{i}= \pm u_{k}$ for $i \neq j$, and $u_{i}^{2}=\alpha_{i} \neq 0$ in $K$. The general element of $C$ is $x=\beta_{1} e+\beta_{2} u_{2}+\cdots+\beta_{8} u_{8}$ and we can define an involution $x \rightarrow \bar{x}$ of $C$ by $\bar{x}=\beta_{1} e-\left(\beta_{2} u_{2}+\cdots+\beta_{8} u_{8}\right)$. If $A=\left(x_{i j}\right)$ is an element of $G$ and $P$ is a diagonal matrix of $G$ with elements in $K$ the mapping $A \rightarrow A^{J}=P\left(\bar{x}_{i j}\right)^{\prime} P^{-1}$ is an involution of $G$. The $J$-symmetric elements of $G$ are closed under the multiplication $(A, B)=2^{-1}(A B+B A)$ and form an exceptional central simple Jordan algebra under this multiplication.

If $P=\left(\pi_{1}, \pi_{2}, \pi_{3}\right)$ for $\pi_{i}$ in $K$, the set of symmetric elements of $G$ are finite linear combinations over $K$ of the elements $e_{i i},\left(\pi_{i} u\right)_{j i}-\left(\pi_{j} u\right)_{i j}$ and $\left(\pi_{i} e\right)_{j i}$ $+\left(\pi_{j} e\right)_{i j}$ where $u$ is any of the $u_{i}$ above. We wish to determine the nature of the multiplication of $A$. From (4) we have that $e_{i i} \cdot e_{i i}=e_{i i}$. Also we obtain $e_{j j} \cdot e_{i i}=0$ from (5) by putting $x=y=e_{i i}$ and $z=e_{j j}$. Identity (5) with $x=e_{i i}-e_{j j}$, $y=e_{i i}, z=\left(\pi_{i} u\right)_{j i}+\left(\pi_{j} \bar{u}\right)_{i j}$ for any of the basal elements $e, u_{2}, \cdots, u_{8}$ gives us $2 e_{j j} \cdot\left[\left(\pi_{i} u\right)_{j i}+\left(\pi_{j} \bar{u}\right)_{i j}\right]=\left(\pi_{i} u\right)_{j i}+\left(\pi_{j} \bar{u}\right)_{i j}=e_{j j}\left[\left(\pi_{i} u\right)_{j i}+\left(\pi_{j} \bar{u}\right)_{i j}\right]+\left(\pi_{i} u\right)_{j i}+\left(\pi_{j} \bar{u}\right)_{i j}$. Now let both $u$ and $v$ be any of the eight basal elements of $C$. If we put $x=e_{j j}$, $y=\left(\pi_{i} u\right)_{j i}+\left(\pi_{j} \bar{u}\right)_{i j}$ and $z=\left(\pi_{i} v\right)_{k i}+\left(\pi_{k} \bar{v}\right)_{i k}$ in $(5)$, we have $\left[\left(\pi_{i} u\right)_{j i}+\left(\pi_{j} \bar{u}\right)_{i j}\right]$ $\cdot\left[\left(\pi_{i} v\right)_{k i}+\left(\pi_{k} \bar{v}\right)_{i k}\right]=2^{-1}\left(\pi_{i} \pi_{j} \bar{u} v\right)_{k j}+2^{-1}\left(\pi_{i} \pi_{k} \bar{v} u\right)_{j k}$. Next put $x=e_{i i}, y=e_{p p}$ and $z=\left(\pi_{p} u\right)_{q p}+\left(\pi_{q} \bar{u}\right)_{p q}$ in (5) we get $e_{i i} \cdot\left[\left(\pi_{p} u\right)_{q p}+\left(\pi_{q} \bar{u}\right)_{p q}\right]=0$. Now put $x$ $=\left(\pi_{i} u\right)_{j i}+\left(\pi_{j} \bar{u}\right)_{i j}, y=\left(\pi_{p} v\right)_{q p}+\left(\pi_{q} \bar{v}\right)_{p q}$ and $z=e_{i i}$ in (5) and obtain $\left[\left(\pi_{j} u\right)_{i j}+\left(\pi_{i} \bar{u}\right)_{j i}\right] \cdot\left[\left(\pi_{p} v\right)_{q p}+\left(\pi_{q} \bar{v}\right)_{p q}\right]=0$. We have shown that all products of basal elements in $A$ agree with their products in $A^{(+)}$except the product $\left[\left(\pi_{i} u\right)_{j i}+\left(\pi_{j} \bar{u}\right)_{i j}\right] \cdot\left[\left(\pi_{i} v\right)_{j i}+\left(\pi_{j} \bar{v}\right)_{i j}\right]$. We will now show that this product has the property. We first show this for $v=e$. Let $x=\left(\pi_{j} e\right)_{i j}+\left(\pi_{i} e\right)_{j i}, y=\left(\pi_{i} e\right)_{k i}$ $+\left(\pi_{k} e\right)_{i k}$ and $z=\left(\pi_{k} \bar{u}\right)_{j k}$ in (5) for $k \neq i, j$. We have $\left[\left(\pi_{k} \pi_{j} e\right)_{i j}+\left(\pi_{k} \pi_{i} e\right)_{j i}\right]$ $\cdot\left[\left(\pi_{j} u\right)_{i j}+\left(\pi_{i} \bar{u}\right)_{j i}\right]+\left[\left(\pi_{j} \pi_{i} e\right)_{k i}+\left(\pi_{k} \pi_{j} e\right)_{i k}\right] \cdot\left[\left(\pi_{i} u\right)_{k i}+\left(\pi_{k} \bar{u}\right)_{i k}\right]=\left[\left(\pi_{i} \pi_{j} e\right)_{k j}\right.$ $\left.+\left(\pi_{i} \pi_{k} e\right)_{j k}\right] \cdot\left[\left(\pi_{j} u\right)_{k j}+\left(\pi_{k} \bar{u}\right)_{j k}\right]$. Letting $x=\left(\pi_{j} u\right)_{i j}+\left(\pi_{i} \bar{u}\right)_{j i}, y=\left(\pi_{i} u\right)_{k i}+\left(\pi_{k} \bar{u}\right)_{i k}$ and $z=\left(\pi_{k} u\right)_{j k}+\left(\pi_{j} \bar{u}\right)_{k j}$ in (5) we have $\pi_{j}\left[\left(\pi_{i} e\right)_{k i}+\left(\pi_{k} e\right)_{i k}\right] \cdot\left[\left(\pi_{i} u\right)_{k i}+\left(\pi_{k} \bar{u}\right)_{i k}\right]$ $+\pi_{k}\left[\left(\pi_{i} e\right)_{j i}+\left(\pi_{j} e\right)_{i j}\right] \cdot\left[\left(\pi_{j} u\right)_{i j}+\left(\pi_{i} \bar{u}\right)_{j i}\right]=\pi_{i}\left[\left(\pi_{k} e\right)_{j k}+\left(\pi_{j} e\right)_{k j}\right] \cdot\left[\left(\pi_{k} u\right)_{j k}+\left(\pi_{j} \bar{u}\right)_{k j}\right]$. These two identities together give us $\left[\left(\pi_{k} e\right)_{j k}+\left(\pi_{i} e\right)_{j i}\right] \cdot\left[\left(\pi_{k} u\right)_{j k}+\left(\pi_{j} \bar{u}\right)_{k j}\right]=0$. Now if $u=v$ in our product we have the square of an element and since $x \cdot x+x \cdot x=x x+x x$, this product agrees with the product in $A^{(+)}$. The remaining case is where both $u$ and $v$ are distinct from $e$ and from each other. Let $w$ 
be the unique basal element such that $u w=v$. Now let $x=\left(\pi_{i} u\right)_{j i}+\left(\pi_{j} \bar{u}\right)_{i j}$, $y=\left(\pi_{k} w\right)_{i k}+\left(\pi_{i} \bar{w}\right)_{i k}$ and $z=\left(\pi_{k} u\right)_{j k}+\left(\pi_{j} \bar{u}\right)_{k j}$ in (5) and we have $\left[\left(\pi_{i} u\right)_{j i}+\left(\pi_{j} \bar{u}\right)_{i j}\right]$ $\cdot\left[\left(\pi_{k} \pi_{j} v\right)_{i j}+\left(\pi_{i} \pi_{k} \bar{v}\right)_{j i}\right]+\left[\left(\pi_{k} u\right)_{j k}+\left(\pi_{j} \bar{u}\right)_{k j}\right] \cdot \pi_{i}\left[\left(\pi_{k} v\right)_{j k}+\left(\pi_{j} \bar{v}\right)_{k j}\right]=0$. Now letting $x=\left(\pi_{i} u\right)_{j i}+\left(\pi_{j} \bar{u}\right)_{i j}, y=\left(\pi_{j} v\right)_{k j}+\left(\pi_{k} \bar{v}\right)_{j k}$ and $z=\left(\pi_{j} e\right)_{k j}+\left(\pi_{k} e\right)_{j k}$ in (5), we obtain $\left[\left(\pi_{i} u\right)_{j i}+\left(\pi_{j} \bar{u}\right)_{i j}\right] \cdot\left[\left(\pi_{k} \pi_{j} v\right)_{i j}+\left(\pi_{k} \pi_{i} \bar{v}\right)_{j i}\right]=\left[\left(\pi_{k} \pi_{i} u\right)_{j k}+\left(\pi_{j} \pi_{i} \bar{u}\right)_{k j}\right]$ $\cdot\left[\left(\pi_{k} v\right)_{j k}+\left(\pi_{j} \bar{v}\right)_{k j}\right]=0$. These two identities give us $\left[\left(\pi_{k} u\right)_{j k}+\left(\pi_{j} \bar{u}\right)_{k j}\right]$ $\cdot\left[\left(\pi_{k} v\right)_{j k}+\left(\pi_{j} \bar{v}\right)_{k j}\right]=0$. We can summarize the above in the following theorem.

TheOREM 2. Let $A$ be an algebra over a field $F$ such that $A^{(+)}$has an ideal $I$ that is a simple, separable, exceptional Jordan algebra. Then $I$ is an ideal of $A$ and the multiplication of $I$ in $A$ agrees with the multiplication of $I$ in $A^{(+)}$.

This theorem together with Theorem 1 gives us:

Theorem 3. Let $A$ be an algebra of class $S J$. Then there is an algebra $G$ that is a direct sum of a semi-simple associative algebra and algebras of threerowed matrices over a Cayley algebra such that $A=B(G, S, T)$.

For each component of $A^{(+)}$that is an exceptional Jordan algebra we define $T_{r} \equiv 0$. This together with the mapping of Theorem 1 defined on the components of $A^{(+)}$that are special gives us the desired mapping $T$.

4. An algebra not of the form $B(G, S, T)$. Let $F$ be a field of characteristic $p \neq 2,3$ with two elements $\alpha$ and $\beta$ that are algebraically independent and transcendental over the prime field of $F$. Let $a$ and $b$ be roots of the irreducible equations $x^{p}-\alpha=0$ and $x^{p}-\beta=0$ over $F$ respectively. The field $K=F(a, b)$ is a nonsimple extension of $F$. The field $K$ is a Jordan algebra under the multiplication $x y$ of the field. We shall now define a second product $x \cdot y$ such that the vector space $K$ is a noncommutative algebra $A$ over $F$ satisfying (1) and having $A^{(+)}=K$. This algebra has a basis consisting of all elements $a^{s} b^{t}$ for $s, t=0,1, \cdots, p-1$. We define

$$
\begin{aligned}
a^{s} b^{t} \cdot a^{m} b^{n} & =a^{s+m} b^{t+n}+(s n-t m)(\alpha-\beta) \\
& =\alpha^{i} \beta^{q} a^{r} b^{i}+(s n-t m)(\alpha-\beta),
\end{aligned}
$$

where $s+m=r+p q, t+n=i+p j$ and $0 \leqq r, i \leqq p-1$. Since $a^{p}=\alpha, b^{p}=\beta$ and the characteristic of $K$ is $p$ we see immediately that this definition holds for all values of $s, t, m$ and $n$. Now let $x=\sum c_{i} a^{s_{i}} b^{t_{i}}, y=\sum d_{j} a^{m_{j}} b^{n_{j}}$. Then $x \cdot y$ $+y \cdot x=x y+y x+\sum c_{i} d_{j}\left(s_{i} n_{j}-t_{i} m_{j}\right)(\alpha-\beta)+\sum c_{i} d_{j}\left(m_{j} t_{i}-n_{j} s_{i}\right)(\alpha-\beta)=2 x y$ and $A^{(+)}=K$. However $A$ is not commutative since $a b \cdot a b^{2}-a b^{2} \cdot a b=2(\alpha-\beta)$ $\neq 0$. Again let $x=\sum c_{i} a^{s i} b^{t_{i}}$. Then $(x \cdot x) \cdot x=(x x) \cdot x=(x x) x+\sum c_{i} c_{j} c_{k}\left(s_{i} t_{k}\right.$ $\left.+s_{j} t_{k}-t_{i} s_{k}-t_{j} s_{k}\right)(\alpha-\beta)=(x x) x$ and

$x \cdot(x \cdot x)=x(x x)+\sum c_{i} c_{j} c_{k}\left(s_{k} t_{i}+s_{k} t_{j}-t_{k} s_{i}-t_{k} s_{j}\right)(\alpha-\beta)=x(x x)=(x x) x=(x \cdot x) \cdot x$.

Therefore Identity (1) holds for the elements of $A$. 
Assume that there exists an algebra $G$ that is a simple associative algebra or an algebra of three-rowed matrices over a Cayley-Dickson algebra such that $A=B(G, S, T)$. If $G$ is of the latter type we see that $T$ must be identically zero and $A$ is commutative. Therefore we shall assume that $G$ is associative. We denote its product by $x \circ y=x y$ for all elements of $K$ we have $x \circ y=y \circ x$ and $x \cdot y=2^{-1}(x \circ y+y \circ x)+(x \circ y-y \circ x) T=x y$ and there is no transformation that bonds $G$ to $A$.

We shall now show that the vector space $K$ is a commutative subalgebra of $G$ under the product $x \circ y$ and therefore $A$ cannot be bonded to $G$ by the above. Recall that $x \circ y+y \circ x=2 x y$ for all $x$ and $y$ in $K$. In particular $x \circ x=x x$ so no confusion should arise from the symbol $x^{2}$. Now $a^{2} \circ b$ $=a \circ(a \circ b)=-(a \circ b) \circ a+2 a(a \circ b)=b \circ a^{2}+2 a(a \circ b)-2 a(b \circ a)=-a^{2} \circ b$ $+4 a(a \circ b)-2 a^{2} b$. So $a^{2} \circ b=2 a(a \circ b)-a^{2} b$. But $a^{2} \circ b=-a \circ b \circ a+2 a(a \circ b)$; therefore $(a \circ b \circ a)=a^{2} b$ and $(a \circ b)^{2}=(a \circ b \circ a \circ b)=a^{2} b \circ b$ and $(b \circ a)^{2}$ $=b \circ a^{2} b$. We also have $2 a b \circ(a \circ b)=a \circ b \circ a \circ b+b \circ a \circ a \circ b=-a \circ b \circ b \circ a$ $+2 a \circ b \circ b \circ a+a^{2} b^{2}=2(a \circ b) \circ a b$. Therefore $a b \circ(a \circ b)=(a \circ b) \circ a b$ $=(a \circ b) a b=a b(a \circ b)$. But $a b(a \circ b)=a b(-b \circ a+2 a b)=-a b(b \circ a)+2 a^{2} b^{2}$ $=-(b \circ a b) \circ a+2 a^{2} b^{2}=a b \circ b \circ a-2 a^{2} b \circ a+2 a^{2} b^{2}$, so $(a \circ b)$ satisfies the identity

$$
x^{2}+(a b) x-2 a^{2} b^{2}=0 .
$$

Now $(a \circ b)^{2}=(-b \circ a+2 a b)^{2}=(b \circ a)^{2}-4 a b(b \circ a)+4 a^{2} b^{2}=-(a \circ b)^{2}$ $+4 a b(a \circ b)-2 a^{2} b^{2}$ or $(a \circ b)$ satisfies the identity $x^{2}-2(a b) x+a^{2} b^{2}=0$. This together with (17) gives us $(a b)(a \circ b)-a^{2} b^{2}=0$. Multiplying by $a^{-1} b^{-1}$ we have $\left(a^{-1} b^{-1}\right) \circ(a b(a \circ b))-a b=a^{-1} b^{-1} \circ a b \circ(a \circ b)-a b=a \circ b-a b=0$. Therefore $a \circ b=a b$ and $G$ is commutative.

5. Subalgebras of $B(G, S, T)$. Let $A$ be an algebra $B(G, S, T)$ where $G$ is an associative algebra.

Theorem 4. Let $G^{\prime}$ be a commutative subalgebra of $G$. Then $G^{\prime} \cap A$ is an associative, commutative subalgebra of $A$.

For if $G^{\prime}$ is a commutative subalgebra of $G$ and $x$ and $y$ are in $G^{\prime} \cap A$ we have $x y-y x=0$. Expressing the product $x \cdot y$ in terms of the mapping $T$ we have $2 x \cdot y=x y+y x+2(x y-y x) T=2 x y$. Therefore products in $A$ of the elements of $G^{\prime} \cap A$ agree with their products in $G$.

Theorem 5. Let $A$ be an algebra of class $S J$ and let $S$ be an associative commutative ideal of $A^{(+)}$. Then $S$ is an associative commutative ideal of $A$.

The components of $G$ corresponding to $S$ can be taken as the associative algebra $S$ since $S=S^{(+)}$. By Theorem $4, S$ is an associative, commutative subalgebra of $A$. Since the components of $A^{(+)}$form orthogonal subspaces of $A$ by Lemma 1 we have that $S$ is an ideal of $A$.

The following theorem is a conjecture of R. Schafer [7]. 
Theorem 6. Let $A$ be a finite power-associative ring without elements of additive order 2, 3 or 5 . If every element a of $A$ satisfies an equation of the form $a^{n(a)}=a, n(a)$ an integer greater than 1 , then $A$ is a vector space sum $A=A_{1}+A_{2}$. The subspace $A_{1}$ is an ideal of $A$ and a direct sum of finite fields. The subspace $A_{2}$ is bonded to $A$ and $A_{2}^{(+)}$is a direct sum of classical 3-dimensional central simple Jordan algebras without nilpotent elements $\neq 0$.

Schafer has proved [7] that under these hypotheses $A^{(+)}$is a direct sum of finite fields and such 3-dimensional Jordan algebras. Since the center of each component of $A^{(+)}$is a finite field, $A^{(+)}$is a separable semi-simple Jordan algebra; hence it is of class $S J$. By Theorem 3 there is an algebra $G$ such that $A=B(G, S, T)$. Write $A=A_{1}+A_{2}$ where $A_{1}^{(+)}$is a direct sum of finite fields and $A_{2}^{(+)}$is a direct sum of 3 -dimensional Jordan algebras. By Theorem 5, $A_{1}^{(+)}=A_{1}$ is a direct sum of finite fields.

\section{BIBLIOGRAPHY}

1. A. A. Albert, $A$ theory of power-associative commutative algebras, Trans. Amer. Math. Soc. vol. 69 (1950) pp. 503-527.

2. - New simple power-associative algebras, Summa Brasiliensis Mathematicas vol. 2 (1951) pp. 1-12.

3. F. D. Jacobson and N. Jacobson, Classification and representation of semi-simple Jordan algebras, Trans. Amer. Math. Soc. vol. 65 (1949) pp. 141-169. 482-494.

4. G. K. Kalisch, On special Jordan algebras, Trans. Amer. Math. Soc. vol. 61 (1947) pp.

5. L. A. Kokoris, New results on power-associative algebras, Trans. Amer. Math. Soc. vol. 77 (1954) pp. 363-373.

6. R. D. Schafer, The exceptional simple Jordan algebras, Amer. J. Math. vol. 70 (1948) pp. 82-94.

7. - A generalization of a theorem of Albert, Proc. Amer. Math. Soc. vol. 4 (1953) pp. $452-455$.

Michigan State University,

East Lansing, Michigan

University of Chicago,

Chicago, Ill. 